\title{
A Prevascularization Strategy Using Novel Fibrous Porous Silk Scaffolds for Tissue Regeneration in Mice with Spinal Cord Injury
}

\author{
Junjie Zhong, ${ }^{1, *}$ Jiaxin $\mathrm{Xu},{ }^{2, *}$ Shijun Lu, ${ }^{3, *}$ Zhifu Wang, ${ }^{1}$ Yongtao Zheng, ${ }^{1}$ \\ Qisheng Tang, ${ }^{1}$ Jianhong Zhu, and Tongming Zhu ${ }^{1}$
}

Spinal cord injury (SCI) represents an extremely debilitating condition for which no efficacious treatment is available. Because spinal cord does not have satisfactory capacity for revascularization after injury, it seems to be a promising way to modulate the lesion environment by reperfusion to promote a regenerative phenotype. Although engineered scaffolds provide a platform to deliver therapeutic cells and neurotrophic factors, slow and insufficient vascularization of large tissue constructs negatively impacts the survival and function of these transplanted cells. In this study, we cocultured our fibrous porous silk scaffold (FPSS) with ADAMTS13overexpressing human umbilical vein endothelial cells (HUVECs) in vitro and transplanted this prevascularized construct into an SCI mouse model. The prevascularized system exhibited a tube-like structure in vitro, promoted vascular infiltration and microvascular network formation after transplantation, and recruited more neural cells to the lesion site. Twenty-eight days later, behavioral analysis showed that locomotor recovery was significantly improved in treated animals compared with control animals. Taken together, our results suggest that the FPSS-HUVECs system promoted neovascularization, guided axon growth at the injury site, and improved the microenvironment. Therefore, this prevascularization system may provide a better therapeutic option for SCI.

Keywords: fibrous porous silk scaffold, prevascularization, vascularization, spinal cord injury, regenerative medicine, tissue engineering

\section{Introduction}

A RECENT LITERATURE review on spinal cord injury (SCI) showed an incidence ranging from 10.4 to 83 cases per million per year and a prevalence of 223-755 per million [1-3]. Over the past 20 years, great emphasis has been placed on cell-mediated regenerative therapies, and exogenous cell transplantation is thought to be an important means of treating SCI. Neuronal function can be improved by applying cells from different sources in SCI, and these cells are not restricted to only exogenous neural stem cells [4-6]. Advances have been achieved, although with considerable challenges. The safety of cell transplantation therapies delivered through multiple routes has been widely confirmed. However, the therapeutic efficacy of these therapies remains unsatisfactory, and the design of studies should be further considered.
In perilesional tissue, there are immature neuronal migration and axonal sprout occurring along neovessels after acute injury in the central nervous system (CNS), suggesting a close relationship between neurogenesis and angiogenesis in regions undergoing active neural tissue reconstruction $[7,8]$. Numerous approaches have linked improvements in functional recovery to increased densities of vascular structure in the spinal cord [9]. Because of the particularity and complexity of SCI, it is difficult for a single strategy to be optimal. Therefore, the combination of revascularization, neuronal regeneration, and manipulated microenvironment are likely necessary [10,11]. Vascular damage after SCI plays an important role in triggering injury-related secondary events, such as severe inflammatory cell infiltration and ischemia. Two days after SCI, the number of blood vessels decreases significantly, and only a few residual blood vessels are observed at the

\footnotetext{
${ }^{1}$ State Key Laboratory for Medical Neurobiology, Department of Neurosurgery, Institutes of Brain Science, Fudan University Huashan Hospital, Shanghai Medical College-Fudan University, Shanghai, China.

${ }^{2}$ Endoscopy Centre and Endoscopy Research Institute, Zhongshan Hospital, Fudan University, Shanghai, China.

${ }^{3}$ The Affiliated Stomatological Hospital of Soochow University, Suzhou Stomatological Hospital, Suzhou, China.

*These authors contributed equally to this work.
} 
injury site. Angiogenesis initiates 3-4 days after SCI, and significant vascular structure is visible a week later [12]. Although a number of studies have shown that endogenous angiogenesis may reach pre-injury vascular levels or even higher, these new vessels do not express the desired phenotype and thus could not achieve satisfactory repair effect. The application of artificial compound containing exogenous vascular endothelial progenitor cells and porous biological scaffolds is an important therapy and a promising strategy for host revascularization and simultaneous tissue regeneration [13].

In recent years, stem cell therapy for SCI has been developed from simple cell transplantation to a combined therapy based on biological scaffolds combining various therapeutic factors with stem cells and has achieved a curative effect [14]. Biological scaffolds, as carriers of cells, provide an artificial microenvironment similar to natural environment for seed cell adhesion, proliferation, and differentiation during tissue reconstruction. In the construction of relatively large artificial tissues, the main obstacle to the survival of seed cells is insufficient vascularization of tissues, which results in limited diffusion of nutrients and oxygen [15]. After the implantation of cellscaffold complex, cells at the core of the complex will undergo apoptosis at an early stage if adequate nutrient supply is not available. Therefore, to overcome this obstacle, an artificial microenvironment needs to be constructed, in which the graft can rapidly integrate to host tissue and achieve vascularization in the early stage after implantation [16,17].

Silk fibroin protein is one of the materials commonly used to construct biological scaffolds, which is a natural protein polymer that can provide a biological structure, deliver angiogenic growth factors efficiently, and replicate some of the features of native angiogenesis $[18,19]$. Moreover, silk fibroin scaffolds can provide an microenvironment for the transplanted cells to be protected from SCI, allowing the cells to proliferate and exert biological effects, and providing bridging support for the cavities after SCI. Recently, we developed a novel kind of fibrous porous silk scaffold (FPSS). Unlike the lamellar or porous structure reported previously, this new three-dimensional silk scaffold consists of a microfibrous structure, which is beneficial for cell migration and axon elongation. This structure can facilitate the movement of seeded cells, diffusion of nutrients, and ingrowth of host tissue after implantation. Prevascularization, another important aspect of angiogenesis therapy, is of great significance for rapid connection with recipient vessels to improve the survival rate of seeded cells after transplantation. In the prevascularization strategy, endothelial cells are preseeded in the scaffolds and incubated several days in vitro to form a microvasculature. The combination of a honeycombed channel structure and prevascularization is considered an efficient way to accelerate vascularization for transplantation.

In this study, we cocultured an FPSS with genetically modified human umbilical vein endothelial cells (HUVECs) and implanted the prevascularized silk scaffolds at sites of SCI in mice. We speculated that a prevascularization strategy may create a microenvironment that is more favorable for angiogenesis and growth of neural fibers, thereby promoting the recovery of spinal cord function.

\section{Materials and Methods}

\section{Preparation and characterization of the FPSS}

Bombyx mori silk was boiled three times in $0.05 \%(\mathrm{w} / \mathrm{v})$ $\mathrm{Na}_{2} \mathrm{CO}_{3}$ solution and then rinsed thoroughly with distilled water to remove sericin. The degummed silk was first dissolved in $98 \%$ formic acid (FA) containing $4 \%$ (w/v) $\mathrm{CaCl}_{2}$, yielding an $8 \%$ (w/v) silk nanofibril-FA solution. The silkFA solution was cast on polystyrene petri dishes to form regenerated silk films after FA evaporation. The prepared silk films were further dissolved in a $9.3 \mathrm{M} \mathrm{LiBr}$ solution at room temperature for $2 \mathrm{~h}$, yielding a $4 \%(\mathrm{w} / \mathrm{v})$ silk- $\mathrm{LiBr}$ solution. This solution was dialyzed against deionized water using a dialysis tube (molecular weight cutoff, 6,000) for 3 days, yielding a $1 \%(\mathrm{w} / \mathrm{v})$ silk nanofibril aqueous solution. The silk nanofibril solution was directly placed at $-20^{\circ} \mathrm{C}$ for $\sim 24 \mathrm{~h}$ to freeze it. The frozen silk nanofibril solution was lyophilized for 3 days and then treated with a $75 \%$ ethanol solution to induce $\beta$-sheet crystallization. The scaffold featured a microfiber structure and was thus termed SF-M.

As a control, a silk porous scaffold was prepared by traditional methods. In brief, the degummed silk was dissolved in a $9.3 \mathrm{M} \mathrm{LiBr}$ solution at $60^{\circ} \mathrm{C}$ for $4 \mathrm{~h}$, yielding a $20 \%$ (w/v) silk-LiBr solution. This solution was dialyzed against deionized water using a dialysis tube (molecular weight cutoff, 6,000) for 3 days, yielding an $\sim 8 \%(\mathrm{w} / \mathrm{v})$ silk aqueous solution. The silk solution was diluted to $1 \%(\mathrm{w} / \mathrm{v})$ with deionized water. The diluted silk solution was frozen at $-20^{\circ} \mathrm{C}$ and lyophilized to form a silk porous scaffold. The silk scaffold was further treated with a $75 \%$ ethanol solution to induce $\beta$-sheet crystallization. The scaffold featured a porous structure and was thus termed SF-P.

Silk scaffolds were cut with a razor blade in liquid nitrogen. The cross-sections were sputtered with gold and then observed with a scanning electron microscope (SEM) (Hitach S-4800, Tokyo, Japan).

Fourier-transform infrared spectrometer (FTIR) spectra were obtained using a NicoLET 5700 spectrometer (Thermo Fisher Scientific, Waltham, MA) in the spectral region of 400$4,000 \mathrm{~cm}^{-1}$, and 64 scans were coded with a resolution of $4 \mathrm{~cm}^{-1}$.

\section{In vitro evaluation of FPSS cocultured with transfected HUVECs}

All the surgical and experimental procedures were reviewed and approved by the Animal and Ethics Review Committee and implemented according to the guidelines established by the Ministry of Health of China.

HUVECs were obtained from ScienCell Biology Technology Company (Shanghai, China), cultured in endothelial basal medium (EBM; ScienCell) devoid of GFs. HUVECs were divided into three groups: the sham group (cells only), control group (transfected cells), and treated group (FPSS seeded with transfected cells). Recombinant DNA (CMVeGFP-IRES-ADAMTS13) was produced as described in a previous study [20]. HUVECs were infected with CMVADAMTS13. The transfection efficiency of these viral vectors has been well documented. Multiplicity of infection was 40. Change to fresh medium was at 24-h after transfection.

The effects of the scaffold on HUVECs were evaluated by plating cells in PLL-coated plates with a small amount of silk scaffold at a density of $1 \times 10^{5}$ cells per well. The cells were 
fixed using $4 \%$ paraformaldehyde, washed three times using phosphate-buffered saline (PBS), and incubated for $45 \mathrm{~min}$ in blocking buffer ( $5 \%$ goat serum, $1 \%$ bovine serum albumin, and $0.2 \%$ Triton $\mathrm{X}-100$ in PBS) at room temperature. After removing the blocking buffer, the cells were incubated overnight with primary antibodies at $4^{\circ} \mathrm{C}$. The following primary antibodies were used: anti-Ve-cad (1:250; Abcam). The next day, the cells were washed using PBS, and a fluorescencelabeled secondary antibody was applied in the dark at room temperature for $1 \mathrm{~h}$. The secondary antibody included Donkey Anti-Rabbit IgG H\&L (Alexa Fluor ${ }^{\circledR}$ 488, 1:1,000; Abcam) and Donkey Anti-Rabbit IgG H\&L (Alexa Fluor 555, 1:1,000; Abcam). The cells were then washed using PBS and stained using a DAPI solution $(1 \mu \mathrm{g} / \mathrm{mL})$, followed by image collection. The cell populations were quantified using ImageJ.

ImageJ (NIH) was used to quantify the viable cells in fluorescence images. All images were analyzed using the same ImageJ macro.

\section{In vivo investigation of the prevascularized FPSS}

Establishment of a hemisected SCI model and transplantation. The experimental protocols were licensed by the Animal Ethics Committees of Fudan University and implemented according to the guidelines established by the Ministry of Health of China. HUVECs $\left(4 \times 10^{6}\right.$ cells in $500 \mu \mathrm{L}$ of medium) were injected into the silk scaffold at a depth of $1.5 \mathrm{~mm}$ using a microsyringe over a period of $5 \mathrm{~min}$. The seeded scaffolds were cultured in expansion medium for 14 days before transplantation.

Adult male C57BL/6 mice with a body weight of $18-22 \mathrm{~g}$ were used for the surgical procedures. The mice were randomized into a control group (laminectomy with hemisection), an FPSS-only group (hemisection with FPSS grafting), and a FPSS-cells group (hemisection with FPSS-cells grafting). There were 12 mice in each group. The mice were anesthetized with sodium pentobarbital $(30 \mathrm{mg} / \mathrm{kg}$ body weight). Before the T9 semi-cut spinal cord column model was established, the T9 vertebral body was exposed by incising the skin and splitting the muscle tissue. Laminectomy was performed at T9 to expose the spinal cord, the spinal cord was hemisected, and a 1-mm column was removed at T9. Then, the spinal cord defect was bridged by (1) a bundle of FPSS or (2) a bundle of FPSS with HUVECs or astrocytes. Furthermore, the muscle layers and skin were sutured individually.

Survival and functional assessment. After surgery, the animals in all four groups were kept in the cages and fed routinely. They were provided the same nursing care, including proper bladder and bowel care. Antibiotics were used to prevent the infection. Until the 28th day after the hemisection, the survival proportions were graphed to illustrate the differences among the four groups.

Every week after SCI model was established, hindlimb locomotor function was assessed according to the BassoBeattie-Bresnahan (BBB) scale. The mice were placed in an open space of $90 \times 120 \mathrm{~cm}$ and allowed to walk freely for 5 min. Two observers, blinded to the groups, were arranged to evaluate locomotor function according to the 21-point BBB scale, with scores ranging from 0 (no hindlimb movement or weight support) to 21 (normal movement).

Harvesting of specimens and immunohistochemical evaluation. Animals were killed at 4 weeks for histological and immunohistochemical evaluation. Parts of the spinal cord from $\mathrm{T} 7$ to 11 were dissected carefully. Then, the specimens from intermediate segment of damage zone were fixed in $4 \%(\mathrm{v} / \mathrm{v})$ formaldehyde, embedded in paraffin wax, and cut into 5 - $\mu \mathrm{m}$-thick sections. The specimens were stained with hematoxylin and eosin to observe and evaluate the structures. For immunohistochemical analysis, the spinal cord sections were stained with primary antibodies against $\mathrm{NF}$ (1:500; Abcam), Ve-cad (1:200; Abcam), and GFAP (1:250; Abcam). The next day, the slides were washed with PBS, and a fluorescence-labeled secondary antibody was added in the dark at room temperature for $1 \mathrm{~h}$. The cells were then washed with PBS and stained with DAPI solution $(2 \mu \mathrm{g} / \mathrm{mL})$.

The anti-Ve-cad-stained sections were observed at a $40 \times$ magnification. Four slides of each animal were counted in each group. Focusing on the areas of high vascular density, the Ve-cad-positive microvascular endothelial cells were counted at a $400 \times$ magnification. All hyperchromatic vascular endothelial cells or endothelial cell clusters separated by connective tissue were regarded as microvascular tissue (Weinder method) [21].

\section{Statistical analysis}

The graphical representations of all data show the mean values and the standard deviation of at least three replicates conducted in triplicate. Analysis of variance (ANOVA) was used for comparisons between experimental groups, and a two-sided $t$-test was used for comparisons between two groups. The differences among all groups were compared using the log-rank test with SPSS software, version 11. A value of $P \leq 0.05$ indicated a significant difference.

\section{Results \\ FPSS was evaluated by SEM and FTIR}

The novel silk scaffold, mainly composed of microfibers, was prepared by a double-dissolving process. The degummed silk was first dissolved in $\mathrm{CaCl} 2-\mathrm{FA}$ solvent to form regenerated silk films. The silk films were further dissolved in a $\mathrm{LiBr}$ solution, yielding a $1 \%$ (w/v) aqueous silk solution containing a nanofibril structure. After lyophilization, the novel silk scaffolds were prepared [22]. In contrast to traditional porous silk scaffolds, the novel silk scaffolds are mainly composed of microfibers. The results from the SEM assay showed that the internal structure of the scaffold is three-dimensional and fibrous, with irregular pores arranged along the longitudinal, transverse direction and with some degree of connectivity (Fig. 1A).

The structure of the silk scaffolds was confirmed by FTIR. As given in Fig. 1B, the as-prepared silk scaffolds ( $a$ and $b$ ) showed absorption peaks at $1,645 \mathrm{~cm}^{-1}$ (amide I) and $1,540 \mathrm{~cm}^{-1}$ (amide II), indicating a mainly random coil and/or silk I conformation. After treatment with $75 \%$ ethanol, the silk scaffolds (c and d) displayed a typical silk II structure with characteristic absorption peaks at $1,630 \mathrm{~cm}^{-1}$ (amide I) and $1,525 \mathrm{~cm}^{-1}$ (amide II). The silk II structure would significantly improve the water stability of silk scaffolds. The double-dissolving process allowed us to prepare a novel microfibrous silk scaffold, and this microfiber structure could 

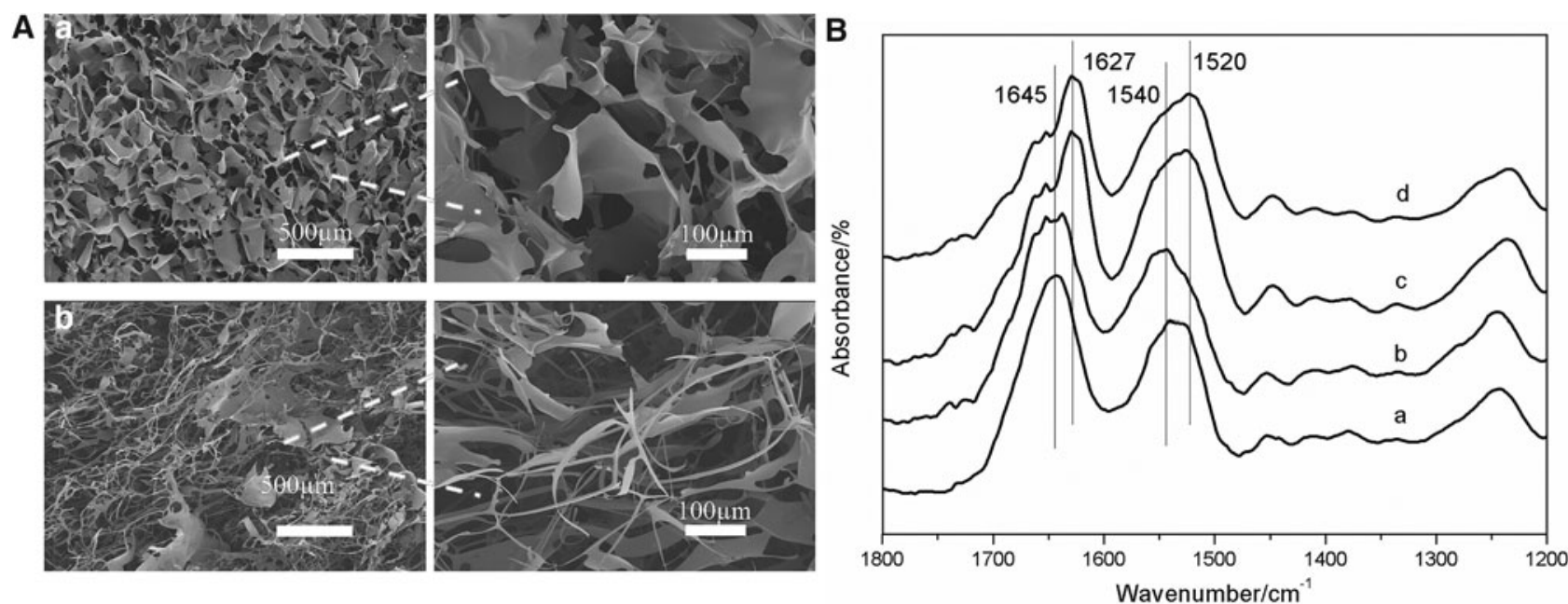

FIG. 1. Dotted line indicates local zoom. (A) SEM images of 3D silk scaffolds. (a) Porous silk scaffolds; (b) fibrous porous silk scaffolds. (B) FTIR spectra of 3D silk scaffolds. Porous silk scaffolds before (a) and after (c) $75 \%$ ethanol treatment; fibrous silk scaffolds before (b) and after (d) $75 \%$ ethanol treatment.
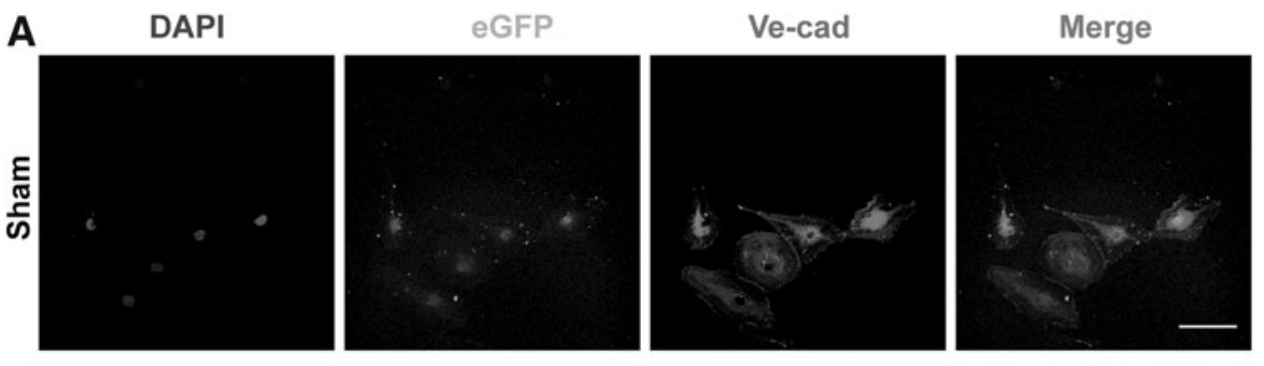

FIG. 2. Effect of FPSS on
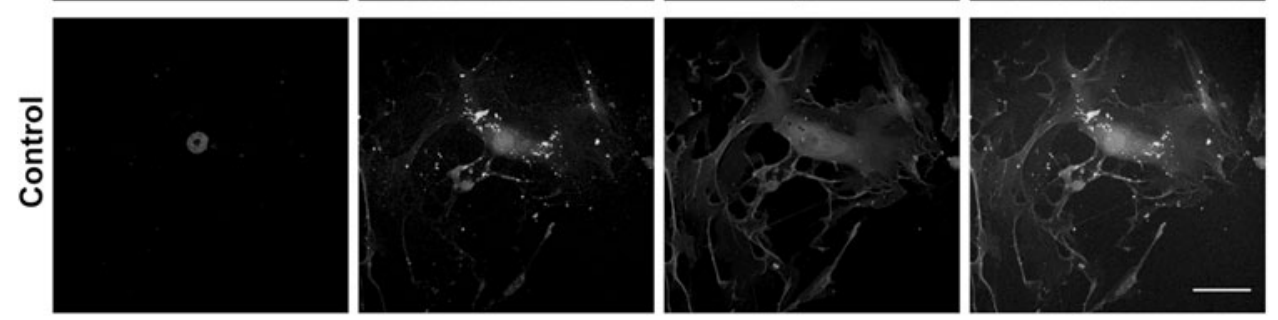

could be found 28 days after coculture. Scale bar: $100 \mu \mathrm{m}$. (B) The FPSS-HUVECtreated group did not show a higher number of $\mathrm{GFP}^{+} /$ Ve-cad ${ }^{+}$cells $(P>0.05)$. FPSS, fibrous porous silk scaffold; HUVEC, human umbilical vein endothelial cell; GFP, green fluorescent protein.
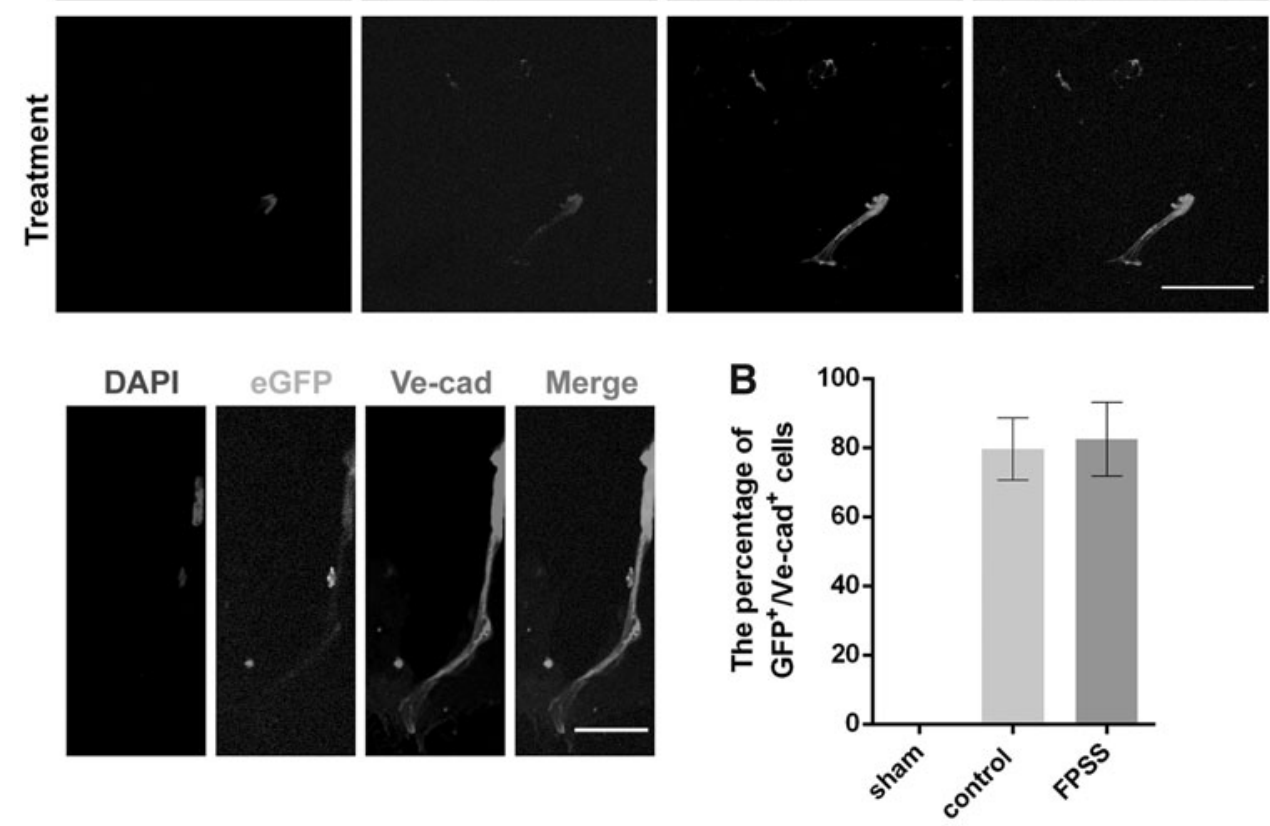
provide a more appropriate microenvironment for cell migration and nerve regeneration.

\section{Effect of porous silk scaffolds on angiogenesis in vitro}

By day 21 after HUVEC transfection, we observed that more $\mathrm{GFP}^{+}$cells with tube-like structures were positive for markers of vascular endothelial cells, such as Ve-cad. The $\mathrm{GFP}^{+} / \mathrm{Ve}-\mathrm{cad}^{+}$cells were quantified at the endpoint after transfection, the transfection efficiency was $82.5 \%$. The FPSS had a robust angiogenic effect compared with that of the control group; the FPSS-HUVEC group did not have a higher number of Ve-cad-positive cells $(P>0.05)$ (Fig. 2B), but Ve-cad-positive vascular lumens could be found at 28 days after coculture (Fig. 2A). These results showed that FPSS could promote angiogenesis in vitro.

\section{FPSS-HUVEC grafts connected the lesion site with less cavity formation}

The implants integrated with host tissue by bridging the rostral and caudal spinal cord stumps. HE staining showed that there was still silk residue at the lesion site acting as a framework 28 days after implantation (Fig. 3). In the control group, most of the silk scaffold was dissolved, and there were numerous cavities in the transected regions. In contrast to other groups, there was tissue continuity across the transection site and integration with host cord stumps in the treatment group. In
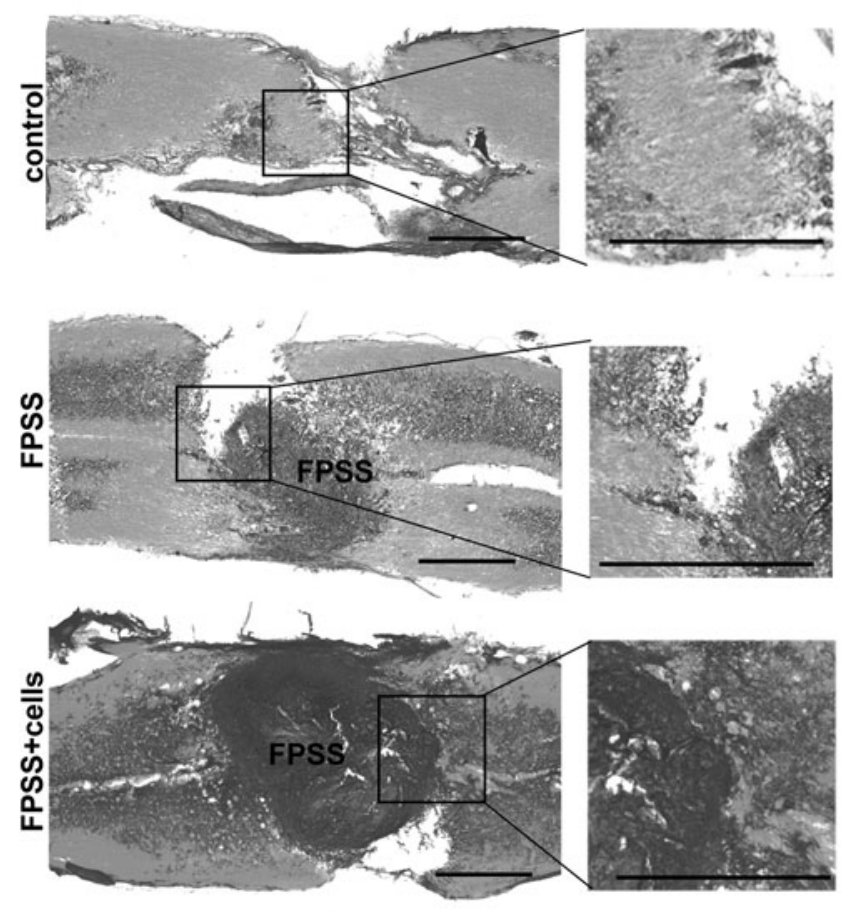

FIG. 3. FPSS-HUVEC grafts connected the lesion site with less cavity formation. HE staining showed that there was silk residue at the lesion site in the control and treated groups 28 days after implantation. The implants integrated with host tissue by bridging the rostral and caudal spinal cord stumps in the treated group. Larger cavities formed and less nerve regeneration occurred in the sham and control groups. Scale bar: $1 \mathrm{~mm}$. HE, hematoxylin and eosin. all groups, scar tissue remained in the transected region, although this feature was obviously lessened in the coculture group. Otherwise, larger cavities formed, and less nerve regeneration occurred in the control group. After observing the images, there were no anomalous cells in any of the groups.

\section{FPSS-HUVEC transplantation enhanced angiogenesis and reduced glial scar formation}

The Ve-cad-positive cells were stained to assess the formation of blood vessels. The microvessel density in the FPSScells group was significantly higher than that in the other groups (Fig. 4A, B) $(P<0.01)$. The results of the microvessel count showed that significantly more blood vessels had formed in the FPSS-cells group than in the other groups (Fig. 4C) $(P<0.01)$, but there was no difference between the control group and FPSS-only group $(P>0.05)$, which means that the scaffold alone could not promote angiogenesis in vivo.

The formation of glial scars was evaluated by the number of $\mathrm{GFAP}^{+}$astrocytes. At 4 weeks after surgery, there were fewer GFAP $^{+}$astrocytes at the injury site in the FPSS-cells group (Figs. 4A and 5A, B). GFAP ${ }^{+}$astrocytes were more abundant in the control group than in the other groups, indicating more pronounced glial scar formation. These results showed that FPSS could inhibit scar formation to a certain extent in vivo $(P>0.05)$, but the results are remarkable when cocultured with transfected HUVECs.

\section{FPSS-HUVECs promoted the formation of a microvascular structure and the ingrowth of neural cells into the site of SCl}

Consistent with the aforementioned results, the number of microvessels at the SCI site was significantly greater in FPSS-cells group than in the other groups at 4 weeks. There were more microvessels at the transplant site and adjacent to the lesion in samples obtained from animals in the FPSS-cells-treated group, even forming a microvascular network. Transplanted cells formed tube-like structures and were surrounded by other tube-like structures (Figs. 5A and 6A). Animals treated with FPSS-HUVECs also showed significantly more abundant neurofilament (NF)-positive cells around the microvessels in the white matter region at the injury site (Fig. 6B) $(P<0.01)$. At 4 weeks after SCI, the FPSS-cells group showed a large amount of NF-positive fibers distributing linearly along the new blood vessels. Some fibers even occasionally grow to the contralateral area of the injury site. These results indicated that the prevascularized FPSS-cells complex effectively enhanced angiogenesis and neural cell ingrowth into the injury site in our model. These findings illustrated that increased angiogenesis and ingrowth of neural fibers into the injury site were associated with certain levels of neural regeneration.

\section{Survival and hindlimb motor function analysis of experimental animals}

After the spinal cord was hemisected, paralysis of the hindlimbs, sensory loss, and dysfunction of the bladder sphincter occurred. A daily intramuscular injection of penicillin was administered for 3 days, followed by weekly 
A

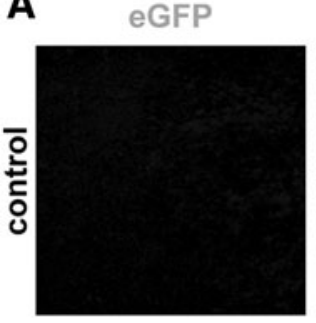

FIG. 4. FPSS-HUVECs enhanced angiogenesis and reduced glial scar formation in vivo. (A) There were fewer GFAP $^{+}$astrocytes at the injury site in the treated group. GFAP $^{+}$astrocytes were more abundant in the sham group than in the control and treated groups. Ve-cad ${ }^{+}$cells were stained to assess the formation of blood vessels. (B) The number of $\mathrm{Ve}-$ $\mathrm{cad}^{+}$cells in the treated group was significantly higher than that in the other groups $(P<0.01)$. (C) The microvessel count results showed that significantly more blood vessels had formed in the treated group than in the control and sham groups $(P<0.01)$. Scale bar: $100 \mu \mathrm{m}$. $* * P<$ $0.01 ; * * * P<0.001$.
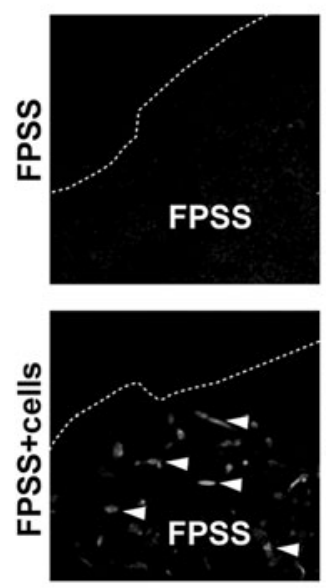
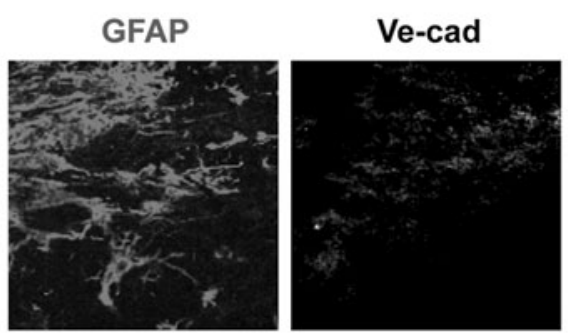

eGFP/GFAPI Ve-cad/DAPI
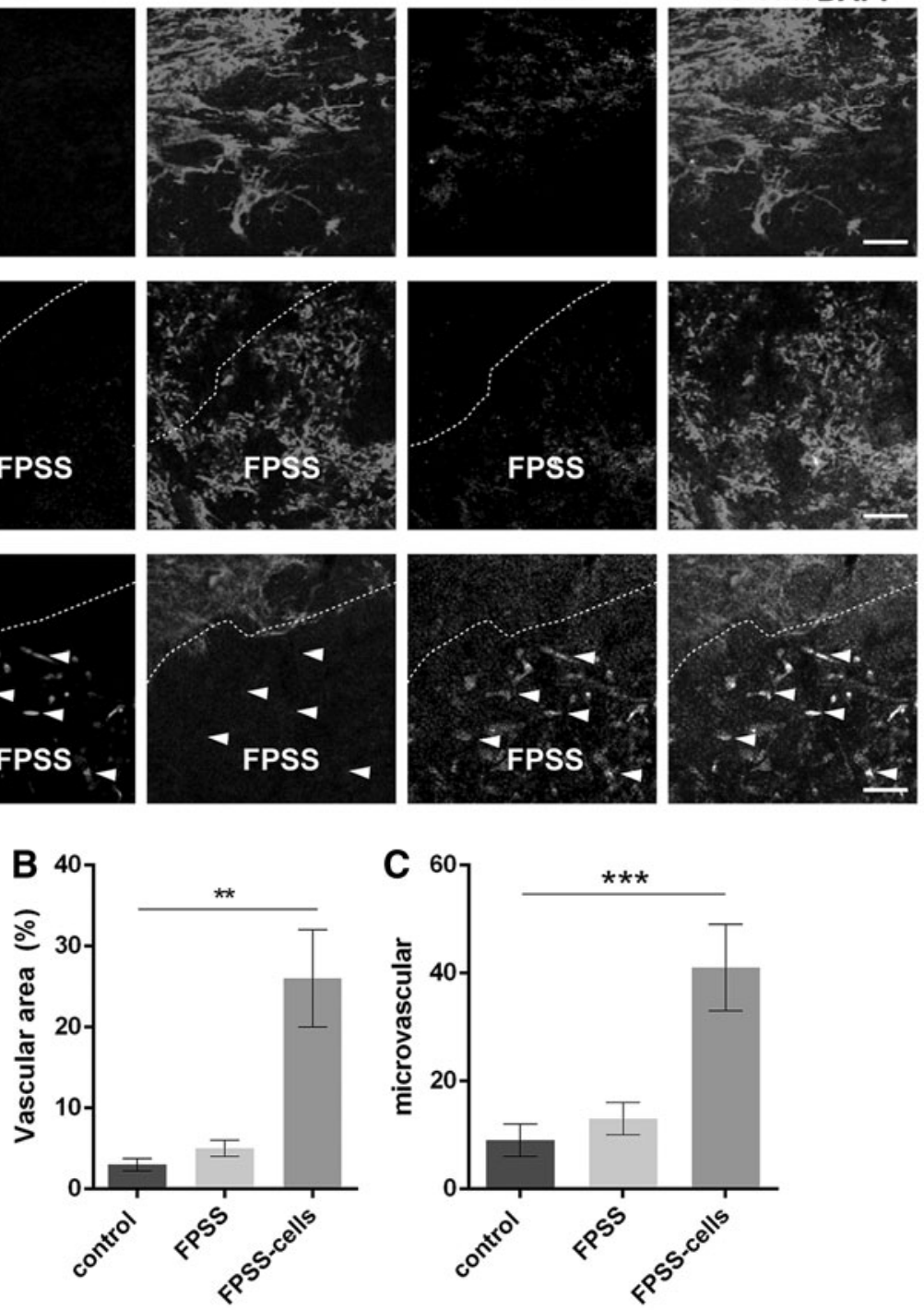

injections. There were no urinary tract or other systemic infections. On day 28 after the hemisection, the survival rate was $100 \%$ in all groups. The survival curve exhibited no significant differences among the three groups.

The BBB scores were used to evaluate the movement of the hindlimbs. Before SCI, all the mice showed normal locomotor performance, with a BBB score of 21 . When the spinal cord was hemisected, the hindlimbs of the animals were immediately paralyzed, with a BBB score of 0 . One week after transplantation, locomotor performance improved gradually in all experimental groups. At the end of the 4th week, the scores of the FPSS-HUVEC group were significantly higher than those of the other groups, and the FPSS-HUVEC group exhibited an average score of $14.78 \pm 1.78$, which was the highest among the groups (Fig. 7) $(P<0.01)$.

\section{Discussion}

A favorable vascular microenvironment is critical to neurologic function recovery after SCI. Microvascular disorder and endothelial cell decrease occur after SCI, and new blood vessels will form in and around the injury center. Although this endogenous angiogenesis response may help improve tissue perfusion, a stronger angiogenesis response is needed to save the injured neural tissue by avoiding further damage and stimulating the regeneration of new tissue, thus facilitating spinal cord repair after injury [23,24]. Biomaterials can aid the modulation of the vascular response following SCI by acting as extracellular matrix (ECM)-like platforms capable of supporting cell growth and proliferation. Biomaterials can be used as ECM-like ecological platforms to support cell growth and proliferation, and improve vascular response regulation after SCI. The capacity of biomaterials to protect seeded cells and therapeutic factors from the harsh microenvironment caused by SCI makes them promising candidates for the development of regenerative medicine [25].

In addition, scaffolds loaded with cells could generate or improve blood perfusion in artificial grafts, thereby improving cell survival and facilitating rapid cell integration with host tissues [26,27]. However, treatment strategies for 

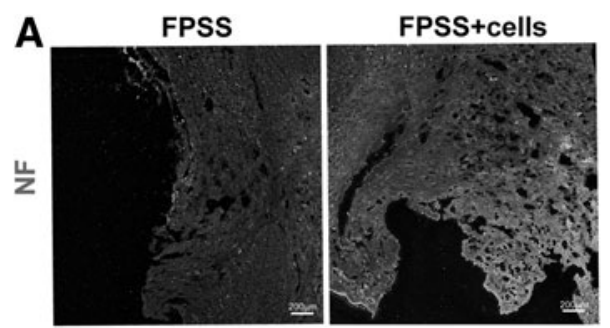
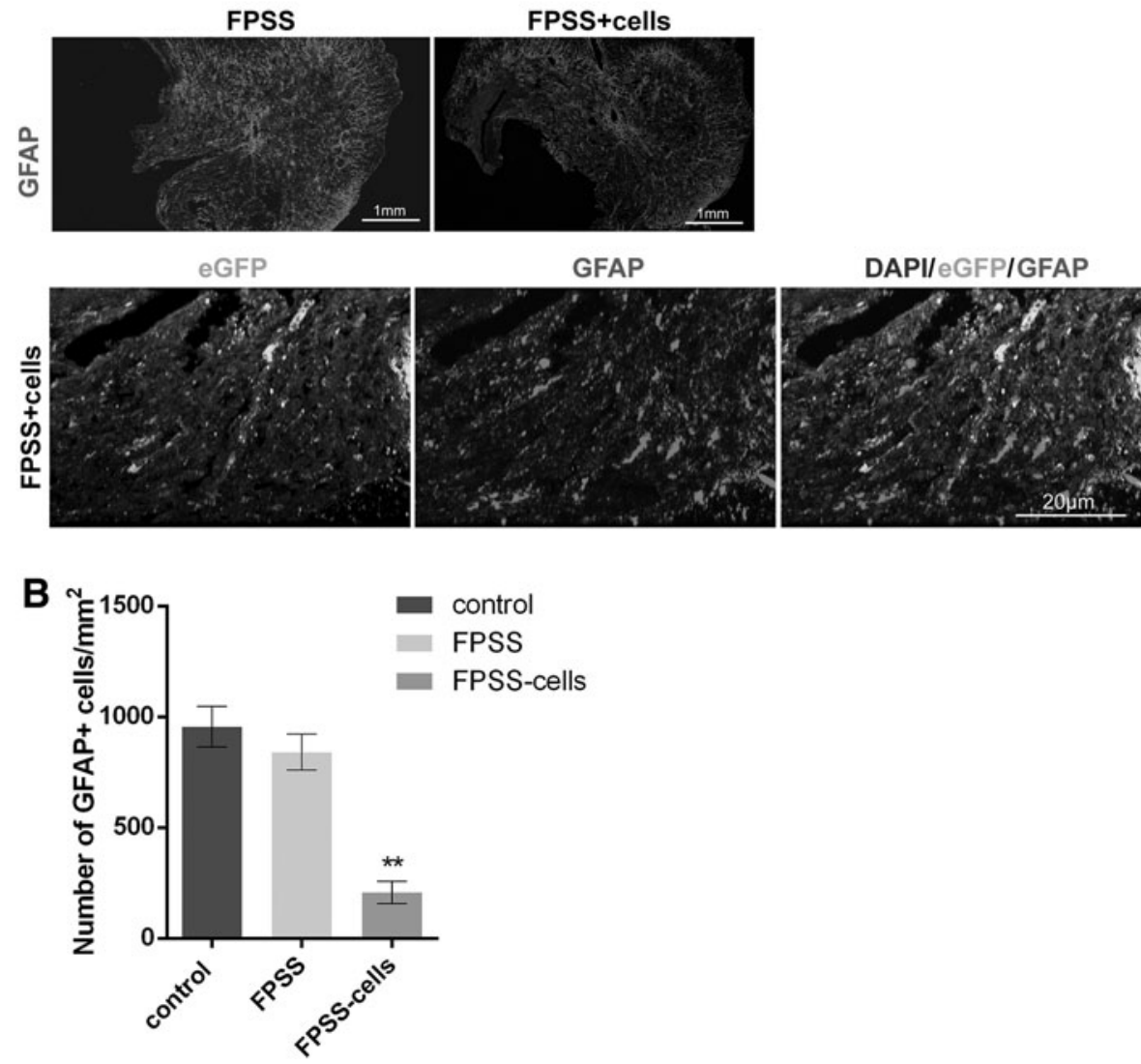

rapid vascularization by using optimized porous silk scaffolds cocultured with genetically modified cells remain a challenge in this field. In this study, we developed a new method to solve this problem. Based on our previous porous silk scaffold experience [22,28], coculture with transfected HUVECs in vitro could form a vascular network to functionally bridge the gap in the injured spinal cord.

Silk fibroin protein biomaterials have been widely used as growth factors and stem cell carriers for nervous tissue regeneration and are considered as scaffold materials of high quality for potential clinical applications [29,30]. In our previous studies, silk nanofibers and porous scaffolds showed good biocompatibility with nerve cells, including neurons, olfactory ensheathing cells, and astrocytes, in vitro and promoted axonal regeneration and myelination in vivo [22,31].

However, the silk nanofibers lacked a 3D structure, and the simple pore structure of the silk scaffold was not interconnected. Recently, the combination of a 3D porous structure and a micro-/nanofibrous structure demonstrated improved tissue neogenesis and collagen matrix formation [32]. In this study, the FPSS presented a fibrous porous
FIG. 5. FPSS-HUVEC transplantation reduced glial scar formation. (A) The formation of glial scars was evaluated by the number of $\mathrm{GFAP}^{+}$astrocytes. There were fewer $\mathrm{GFAP}^{+}$astrocytes and more $\mathrm{NF}^{+}$cells at the injury site in the FPSS-cells group than in other groups. $\mathrm{GFP}^{+}$transplanted cells formed tube-like structures at the injury site. (B) The FPSS-HUVEC group showed less numbers of $\mathrm{GFAP}^{+}$astrocytes than the control groups $(P<0.01)$. $* * P<0.01$. structure, which is conducive to cell adhesion and sufficient exchange of oxygen and nutrients. In addition, the interconnected pore structure is very similar to the original biological tissue and can provide a good microenvironment for regeneration, bridge spinal cord defects, and promote functional recovery.

In the field of regenerative medicine, prevascularization with vascular endothelial cells in artificial tissue structures has been well studied. The advantage of this strategy is that faster and richer host vascular connections can be established for the prevascular grafts after transplantation, thus providing essential biological nutrients for the reconstructed tissue [33]. However, the main barrier to HUVECs survival and functioning is the lack of adequate oxygen and nutrients. The fibrous porous silk structure not only facilitates the migration and distribution of cells during coculture, but also improves the transport of nutrients to the interior of scaffolds, thus facilitating graft and host tissue fusion.

More importantly, the fibrous porous structure allowed cocultured HUVECs to form tubular structures in vitro, which retained their original structure and function after transplantation in vivo. When implanting the scaffolds 

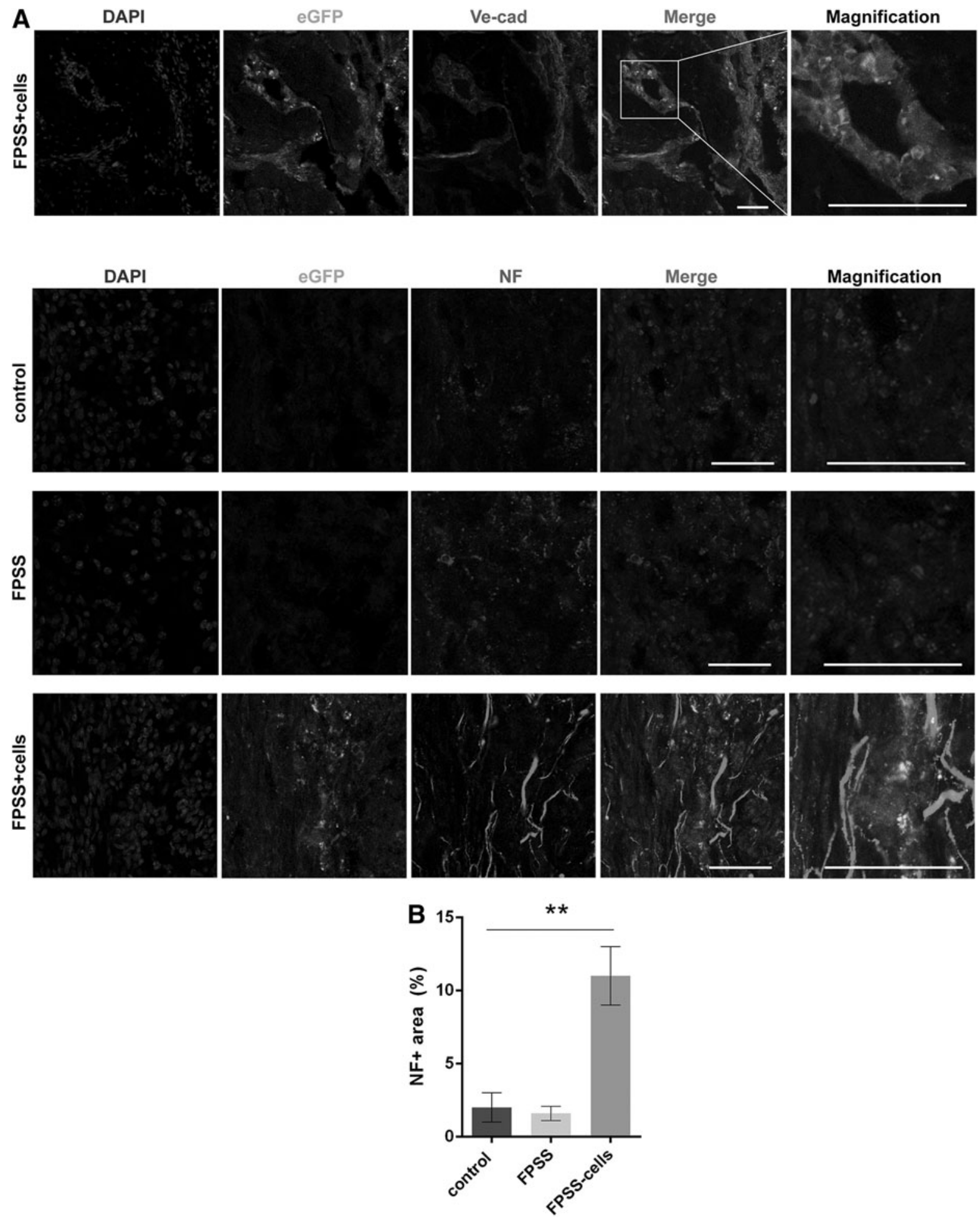

FIG. 6. FPSS-HUVECs promoted microvascular structure formation and neural cell ingrowth into the injury site. (A) In tissues from the treated group, microvessels were abundant at the injection site and formed tube-like structures. Massive NFpositive fibers aligned with blood vessels and traversed the injury epicenter. (B) The FPSS-HUVEC-treated group showed significantly greater numbers of $\mathrm{NF}^{+}$cells than the control and sham groups $(P<0.01)$. Scale bar: $100 \mu \mathrm{m}$. $* * P<0.01$.

in vivo without cocultured cells, the host cells could not migrate into the scaffold using the small holes, which indicated a combined ability of the honeycombed porosity and prevascularization to promote rapid vascularization in vivo. The formation of glial scars at the local site was an obstacle to the extension of regenerated neural fibers. Tissue engineering strategies also aim to replace the cyst/ scar with functional tissue. The regenerated nerve fibers extended in an ordered direction along the tube in the scaffolds in vivo, and there were more ordered NF-positive 


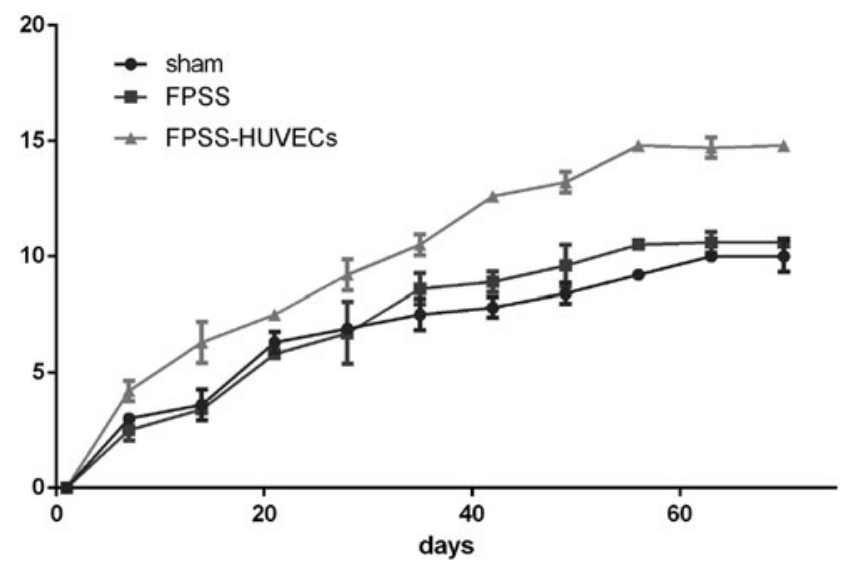

FIG. 7. The BBB scores of the FPSS-HUVEC group were significantly higher than those of the other groups $(P<0.01)$. Compared with the control groups, the BBB scores of the FPSS-TA (transfected astrocyte) group did show any significant difference $(P>0.05)$. BBB, BassoBeattie-Bresnahan.

neural fibers and fewer GFAP-positive cells at the injury site in the FPSS-HUVECs group than in other groups. The largest area of Ve-cad-positive cells was also found in the FPSS-HUVECs group, indicating more pronounced capillary formation. Our immunohistochemistry suggests the FPSS-HUVECs delivery system is capable of promoting neural regeneration and the formation of supporting capillaries. It might create a more suitable microenvironment at the injury site, providing the regenerating neural fibers with linear guidance and promoting capillary formation.

Neural progenitor cells are endogenous to the ependymal regions of the central canal of the spinal cord [4]. SCI can trigger an endogenous neurogenesis response in which stem cells proliferate and migrate from the central canal to the damage area, differentiating into neurons, oligodendrocytes, and astrocytes. These differentiated cells play an important role in tissue reconstruction after SCI [34]. There are several challenges for exogenous stem cell transplantation, including immune rejection, tumor formation, and ethical concerns. However, endogenous stem cells do not have these problems; hence, several strategies aiming at activating progenitor cells have been investigated in SCI. The relationship between angiogenesis and neurogenesis has been explored in many studies.

The paucity of physical guidance disrupts nerve regeneration through the site of SCI limiting successful traversal [35]. Paracrine factors produced by new blood vessels induce immature neuronal cells to migrate to the damaged area, and promote the repair of damaged tissues after SCI. Similarly, in this study we find that enhanced angiogenesis is closely related to increased neurogenesis at the spinal cord lesion site. We found ingrowth of neural fibers into the lesion site, and these cells were closely adjacent to the neovascular. At the same time, no significant immunosuppression was found in our study. The new microvascular network may serve as an effective frame structure supporting growth/regrowth across the lesion site.

In the field of neuroscience, research on cell-mediated regenerative therapy for SCI is still in the preliminary stage.
Although the desire to promote artificial scaffolds with multiple types of stem cells for SCI is extremely strong, current knowledge about the mechanisms of this therapy is poor, so further study should be focused on a new strategy of scaffold-cell combination and the mechanism of action of trophic factors.

\section{Author Disclosure Statement}

No competing financial interests exist.

\section{Funding Information}

This study was supported by the National Key R\&D Program of China (2017YFC0110304 and 2018YFA0107900).

This study was also supported by the National Natural Science Foundation of China (81601069 and 31771491).

\section{References}

1. Wyndaele M and JJ Wyndaele. (2006). Incidence, prevalence and epidemiology of spinal cord injury: what learns a worldwide literature survey? Spinal Cord 44:523-529.

2. Eckert MJ and MJ Martin. (2017). Trauma: spinal cord injury. Surg Clin North Am 97:1031-1045.

3. Ma F, R Li, H Tang, T Zhu, F Xu and J Zhu. (2019). Regulation of autophagy in mesenchymal stem cells modulates therapeutic effects on spinal cord injury. Brain Res 1721:146321.

4. Zhu T, Q Tang, H Gao, Y Shen, L Chen and J Zhu. (2014). Current status of cell-mediated regenerative therapies for human spinal cord injury. Neurosci Bull 30:671-682.

5. Guo S, L Wang, Y Xie, X Luo, S Zhang, L Xiong, H Ai, Z Yuan and J Wang. (2019). Bibliometric and visualized analysis of stem cells therapy for spinal cord injury based on Web of Science and CiteSpace in the recent 20 years. World Neurosurg 132:e246-e258.

6. Rocha LA, RA Sousa, DA Learmonth and AJ Salgado. (2018). The role of biomaterials as angiogenic modulators of spinal cord injury: mimetics of the spinal cord, cell and angiogenic factor delivery agents. Front Pharmacol 9:164.

7. Hachem LD, AJ Mothe and CH Tator. (2017). Positive modulation of AMPA receptors promotes survival and proliferation of neural stem/progenitor cells from the adult rat spinal cord. Stem Cells Dev 26:1675-1681.

8. Wang J, W Zou, J Ma and J Liu. (2019). Biomaterials and gene manipulation in stem cell-based therapies for spinal cord injury. Stem Cells Dev 28:239-257.

9. Sinescu C, F Popa, VT Grigorean, G Onose, AM Sandu, M Popescu, G Burnei, V Strambu and C Popa. (2010). Molecular basis of vascular events following spinal cord injury. J Med Life 3:254-261.

10. Zhang W, LS Wray, J Rnjak-Kovacina, L Xu, D Zou, S Wang, M Zhang, J Dong, G Li, DL Kaplan and X Jiang. (2015). Vascularization of hollow channel-modified porous silk scaffolds with endothelial cells for tissue regeneration. Biomaterials 56:68-77.

11. Abdeen AA and K Saha. (2017). Manufacturing Cell Therapies Using Engineered Biomaterials. Trends Biotechnol 35:971-982.

12. Bakshi A, O Fisher, T Dagci, BT Himes, I Fischer and A Lowman. (2004). Mechanically engineered hydrogel scaffolds for axonal growth and angiogenesis after transplantation in spinal cord injury. J Neurosurg Spine 1:322-329. 
13. Browne S and A Pandit. (2017). Engineered systems for therapeutic angiogenesis. Curr Opin Pharmacol 36:34-43.

14. Akar B, B Jiang, SI Somo, AA Appel, JC Larson, KM Tichauer and EM Brey. (2015). Biomaterials with persistent growth factor gradients in vivo accelerate vascularized tissue formation. Biomaterials 72:61-73.

15. Laschke MW, Y Harder, M Amon, I Martin, J Farhadi, A Ring, N Torio-Padron, R Schramm, M Rucker, et al. (2006). Angiogenesis in tissue engineering: breathing life into constructed tissue substitutes. Tissue Eng 12:20932104.

16. Lutolf MP, PM Gilbert and HM Blau. (2009). Designing materials to direct stem-cell fate. Nature 462:433-441.

17. Lovett M, K Lee, A Edwards and DL Kaplan. (2009). Vascularization strategies for tissue engineering. Tissue Eng Part B Rev 15:353-370.

18. Omenetto FG and DL Kaplan. (2010). New opportunities for an ancient material. Science 329:528-531.

19. Vera-Sanchez M, S Aznar-Cervantes, E Jover, D GarciaBernal, RE Onate-Sanchez, D Hernandez-Romero, JM Moraleda, M Collado-Gonzalez, FJ Rodriguez-Lozano and JL Cenis. (2016). Silk-Fibroin and graphene oxide composites promote human periodontal ligament stem cell spontaneous differentiation into osteo/cementoblast-like cells. Stem Cells Dev 25:1742-1754.

20. Xu H, Y Cao, X Yang, P Cai, L Kang, X Zhu, H Luo, L Lu, L Wei, et al. (2017). ADAMTS13 controls vascular remodeling by modifying VWF reactivity during stroke recovery. Blood 130:11-22.

21. Ma F, F Xu, R Li, Y Zheng, F Wang, N Wei, J Zhong, Q Tang, T Zhu, Z Wang and J Zhu. (2018). Sustained delivery of glial cell-derived neurotrophic factors in collagen conduits for facial nerve regeneration. Acta Biomater 69:146-155.

22. Lu S, P Wang, F Zhang, X Zhou, B Zuo, X You, Y Gao, H Liu and H Tang. (2015). A novel silk fibroin nanofibrous membrane for guided bone regeneration: a study in rat calvarial defects. Am J Transl Res 7:2244-2253.

23. Ng MT, AT Stammers and BK Kwon. (2011). Vascular disruption and the role of angiogenic proteins after spinal cord injury. Transl Stroke Res 2:474-491.

24. Lin XY, BQ Lai, X Zeng, MT Che, EA Ling, W Wu and YS Zeng. (2016). Cell transplantation and neuroengineering approach for spinal cord injury treatment: a summary of current laboratory findings and review of literature. Cell Transplant 25:1425-1438.

25. Sensharma P, G Madhumathi, RD Jayant and AK Jaiswal. (2017). Biomaterials and cells for neural tissue engineering: current choices. Mater Sci Eng C Mater Biol Appl 77: 1302-1315.

26. Bianco P and PG Robey. (2001). Stem cells in tissue engineering. Nature 414:118-121.

27. Rauch MF, SR Hynes, J Bertram, A Redmond, R Robinson, C Williams, H Xu, JA Madri and EB Lavik. (2009). Engineering angiogenesis following spinal cord injury: a coculture of neural progenitor and endothelial cells in a degradable polymer implant leads to an increase in vessel density and formation of the blood-spinal cord barrier. Eur J Neurosci 29:132-145.
28. Zhang F, B Zuo, Z Fan, Z Xie, Q Lu, X Zhang and DL Kaplan. (2012). Mechanisms and control of silk-based electrospinning. Biomacromolecules 13:798-804.

29. Altman GH, F Diaz, C Jakuba, T Calabro, RL Horan, J Chen, H Lu, J Richmond and DL Kaplan. (2003). Silkbased biomaterials. Biomaterials 24:401-416.

30. Nazarov R, HJ Jin and DL Kaplan. (2004). Porous 3-D scaffolds from regenerated silk fibroin. Biomacromolecules 5:718-726.

31. Shen Y, Y Qian, H Zhang, B Zuo, Z Lu, Z Fan, P Zhang, F Zhang and C Zhou. (2010). Guidance of olfactory ensheathing cell growth and migration on electrospun silk fibroin scaffolds. Cell Transplant 19:147-157.

32. Haggerty AE, MM Marlow and M Oudega. (2017). Extracellular matrix components as therapeutics for spinal cord injury. Neurosci Lett 652:50-55.

33. Dray C, G Rougon and F Debarbieux. (2009). Quantitative analysis by in vivo imaging of the dynamics of vascular and axonal networks in injured mouse spinal cord. Proc Natl Acad Sci U S A 106:9459-9464.

34. Siddiqui AM, M Khazaei and MG Fehlings. (2015). Translating mechanisms of neuroprotection, regeneration, and repair to treatment of spinal cord injury. Prog Brain Res 218:15-54.

35. Guvendiren M and JA Burdick. (2013). Engineering synthetic hydrogel microenvironments to instruct stem cells. Curr Opin Biotechnol 24:841-846.

Address correspondence to: Tongming Zhu State Key Laboratory for Medical Neurobiology Department of Neurosurgery Institutes of Brain Science Fudan University Huashan Hospital Shanghai Medical College-Fudan University 12 Urumqi Zhong Road Shanghai 200040 China

E-mail: ztm1911@163.com

Jianhong Zhu

State Key Laboratory for Medical Neurobiology

Department of Neurosurgery Institutes of Brain Science Fudan University Huashan Hospital Shanghai Medical College-Fudan University 12 Urumqi Zhong Road Shanghai 200040 China

E-mail: jzhu@fudan.edu.cn

Received for publication September 25, 2019 Accepted after revision February 18, 2020

Prepublished on Liebert Instant Online February 21, 2020 